\title{
Identification of the novel Kawasaki 2014 GII.17 human norovirus strain in Italy, 2015
}

\author{
MC Medici ${ }^{1}$, F Tummolo ${ }^{1}$, A Calderaro ${ }^{1}$, M Chironna ${ }^{2}$, GM Giammanco ${ }^{3}$, S De Grazia ${ }^{3}$, MC Arcangeletti ${ }^{1}$, F De Conto ${ }^{1}$, C \\ Chezzi ${ }^{1}$, V Martella $^{4}$ \\ 1. Unit of Microbiology and Virology, Department of Clinical and Experimental Medicine, University of Parma, Parma, Italy \\ 2. Department of Biomedical Science and Human Oncology, University of Bari Aldo Moro-Policlinico, Bari, Italy \\ 3. Department of Health Promotion Sciences and Mother and Child Care 'G. D'Alessandro', University of Palermo, Palermo, Italy \\ 4. Department of Veterinary Medicine, University of Bari Aldo Moro, Bari, Italy
}

Correspondence: Maria Cristina Medici (mariacristina.medici@unipr.it)

Medici MC, Tummolo F, Calderaro A, Chironna M, Giammanco GM, De Grazia S, Arcangeletti MC, De Conto F, Chezzi C, Martella V. Identification of the novel

Kawasaki 2014 Gll.17 human norovirus strain in Italy, 2015. Euro Surveill. 2015;20(35):pii=30010. D0I: http://dx.doi.org/10.2807/1560-7917.ES.2015.20.35.30010

Article submitted on 24 August 2015 / accepted on 03 September 2015 / published on 03 September 2015

Surveillance of noroviruses in Italy identified the novel GII.17 human norovirus strain, Kawasaki 2014, in February 2015. This novel strain emerged as a major cause of gastroenteritis in Asia during 2014/15, replacing the pandemic GII.4 norovirus strain Sydney $\mathbf{2 0 1 2}$, but being reported only sporadically elsewhere. This novel strain is undergoing fast diversification and continuous monitoring is important to understand the evolution of noroviruses and to implement the future strategies on norovirus vaccines.

During the winter season 2014/15, a novel GII.P17GII.17 norovirus (NoV) strain emerged in Asian countries [1-4]. Since its emergence, this novel NoV strain, named Kawasaki 2014, has replaced the previously dominant Gll.4 genotype Sydney 2012 variant in Asia, and it has been detected in a limited number of cases on other continents [1-5]. This epidemiological trend is also reflected in the GenBank database, with the vast majority of the Kawasaki 2014 Gll.17 NoV sequences generated in studies from the Asian continent.

Here we report the detection of the Kawasaki 2014 GII.17 strain during the 2014/15 winter season in Italy. As sequence information on Kawasaki 2014 Gll.17 NoVs detected outside the Asian continent is limited [5], we determined the sequence of a large portion of the genome, including the full-length capsid gene of the GII.17 Kawasaki NoV strain circulating in Italy, and analysed the virus sequence with similar GII.17 NoV sequences available in the GenBank database.

\section{Genotyping}

The NoV genome contains three open reading frames (ORFs). ORF1 encodes non-structural proteins including the RNA-dependent RNA polymerase (RdRp), while ORF2 and $\mathrm{ORF}_{3}$ encode the major capsid protein $\mathrm{VP}_{1}$ and a minor structural protein $\mathrm{VP}_{2}$, respectively [6].
NoVs are classified in at least six genogroups, GI to GVI [6]. NoV genogroups are further divided in various genotypes based on differences in the $R d R p$ region (polymerase genotype, or pol type) and in the VP1 (capsid genotype, or cap type) [7]. NoV genotyping was performed using standardised sequence analysis webbased tools developed and maintained by the NoroNet [8].

\section{Surveillance of noroviruses in Italy}

The Italian Study Group for Enteric Viruses (ISGEV; http://isgev.net) monitors the epidemiology of enteric viruses in children through hospital-based surveillance. A subset of about half of the NoV-positive samples is systematically genotyped in both region $A$ (ORF1, RdRp) and region C (ORF2, capsid). From September 2014 to March 2015, NoV prevalence was 12\% (137/1,144) and NoVs were typed in 81 cases (59\%). GII.P17-GII.17 NoV strains were detected in two sporadic cases of acute severe gastroenteritis in young children hospitalised in February 2015 in two distinct Italian regions.

\section{Sequence analysis}

Upon direct sequencing of the RT-PCR amplicons, the two strains, PR668/2015/ITA and BA603-6/2015/ ITA, were found to be identical in the short diagnostic regions $A$ and $C$. We determined the sequence of a large portion (3.2 kb) of the genome at the 3' end for strain PR668/2015/ITA. Viral RNA was extracted from $140 \mu \mathrm{l}$ of stool suspension using the QIAmp viral RNA kit (Qiagen, GmbH, Hilden, Germany). A 3'-rapid amplification of cDNA ends (RACE)-PCR) protocol was used to generate the $3.2-\mathrm{kb}$ amplicon encompassing the 3 ' end of ORF1, the full-length ORF2 and ORF3, and the 3 ' untranslated region (UTR) until the poly (A) tail, using the reverse primer $\mathrm{VN}_{3} \mathrm{~T}_{20}$ [9] and the forward primer JV12Y [10]. The RACE product was cloned and the 


\section{FIGURE 1}

Phylogenetic analysis based on partial ORF1, full ORF2 and full ORF3 sequences of GII.17 NoV, Italy, February 2015
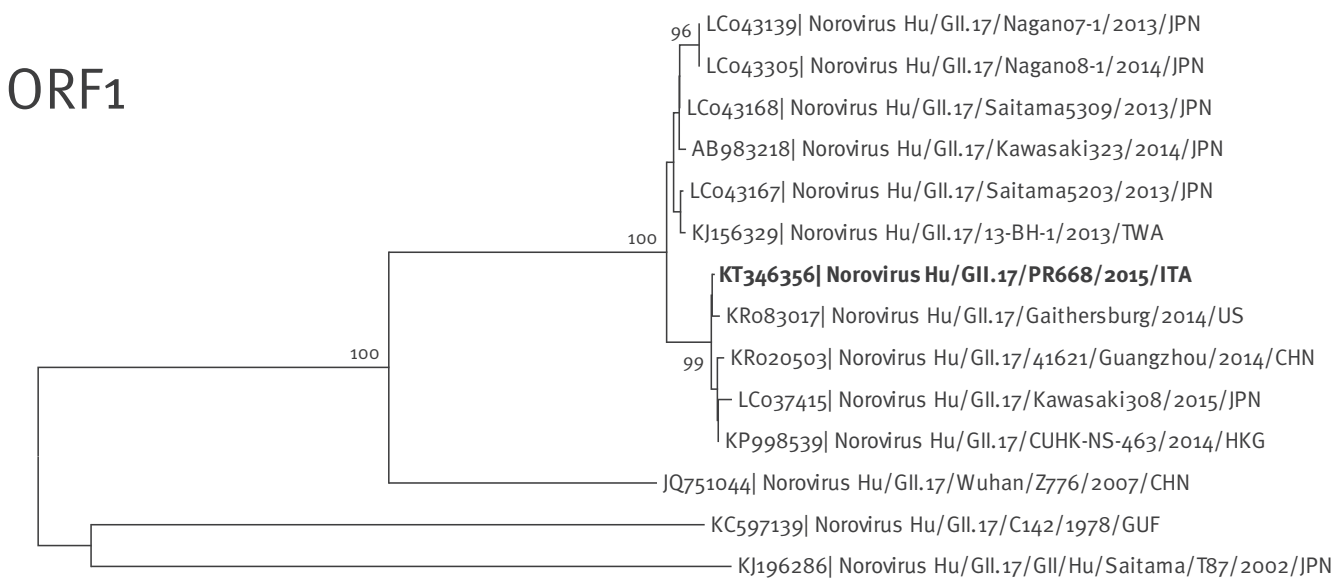

\section{ORF2}

100

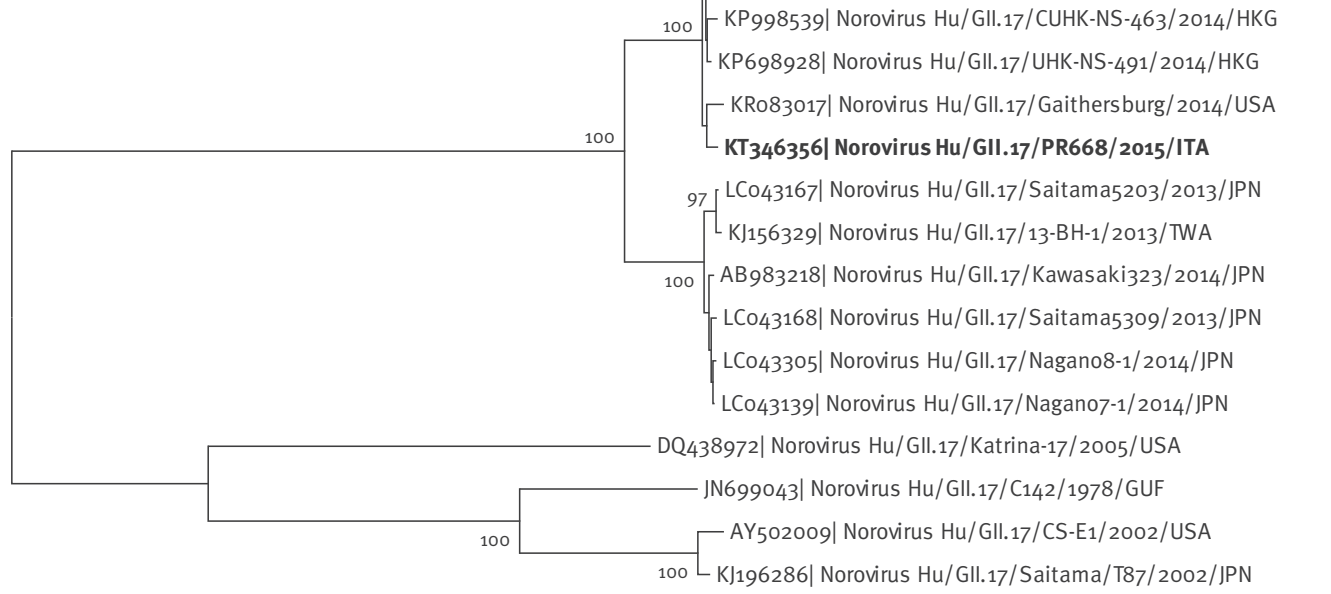

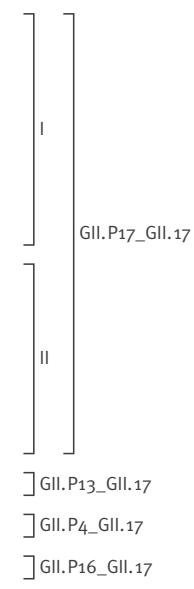

GII.P17_GII.17

]GII.P13_GII.17

] GII.P4_GII.17

GII.P16_GII.17

0.02

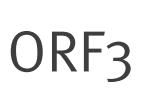

$94-\mathrm{KJ} 156329 \mid$ Norovirus Hu/GIl.17/13-BH-1/2013/TWA

LC043167| Norovirus Hu/GIl.17/Saitama5203/2013/JPN

LC043168| Norovirus Hu/GIl.17/Saitama5309/2013/JPN

AB983218| Norovirus Hu/GII.17/Kawasaki323/2014/JPN

LC043139| Norovirus Hu/GIl.17/Nagano7-1/2014/JPN

${ }_{1008}$ LC043305| Norovirus Hu/GII.17/Nagano8-1/2014/JPN
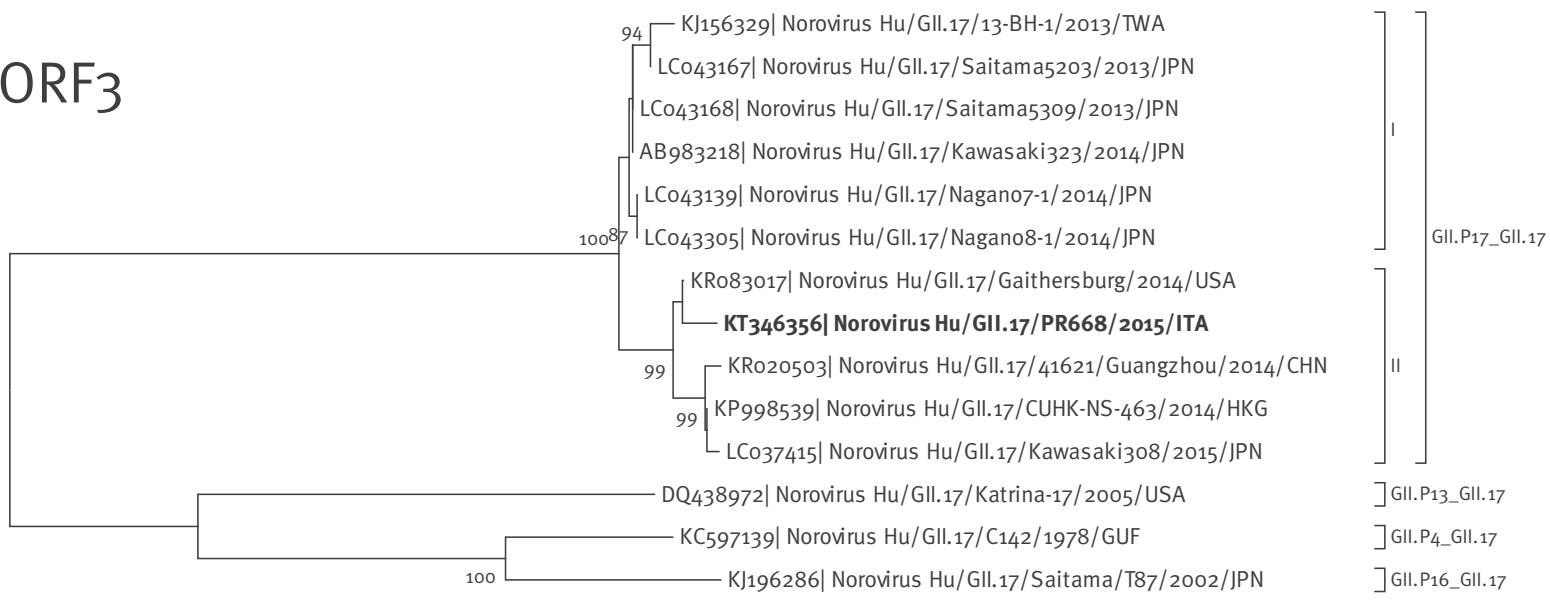

0.02

The Italian GII.P17_GII.17 strain is indicated in bold. Trees were built with the maximum-likelihood method, and bootstrapped with 1,00o repetitions. Bootstrap values $>80 \%$ are indicated. The scale bar indicates the number of nucleotide substitutions per site. 


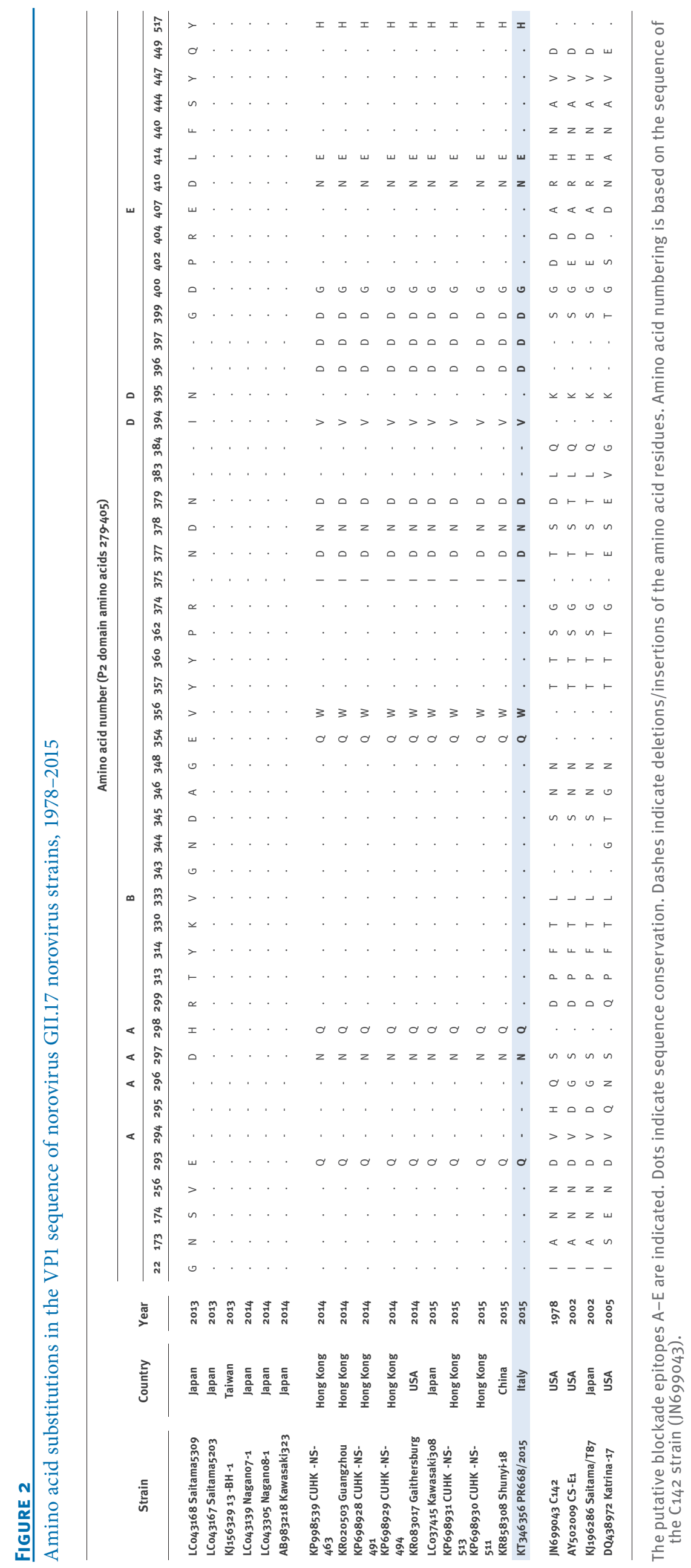


sequence was determined. Phylogenetic analysis was performed using MEGA v. 6.0 [11].

The 3.2-kb sequence of the Italian NoV GII.P17-GII.17 strain has been deposited in GenBank under accession number $\mathrm{KT}_{346356}$. The partial sequence of ORF1 (807 nt), and the full-length sequences of ORF2 $(1,621$ nt) and ORF3 (849 nt) of strain PR668/2015/ITA were analysed with NoV GII.P17-GII.17 sequences available in the GenBank database (Figure 1).

The topology of the trees in the multi-target phylogenetic analysis was conserved, with the GII.P17-GII.17 Kawasaki 2014 NoV forming a monophyletic branch and further segregating into two genetic subclades. The first subclade containing the Italian PR668/2015/ ITA strain clustered with GII.P17-GII.17 NoVs detected in China and Hong Kong during 2014 and 2015, and was genetically related (99.9\%) to a GII.P17-GII.17 strain detected in the United States (US) in November 2014. The second subclade included GII.P17-GII.17 NoV detected in Japan and Taiwan during 2013 and 2014. The viruses of the two subclades showed a moderate degree of nucleotide and amino acid divergence in the ORF2 and ORF3 sequences (1-1.9\% nucleotide and $0-0.4 \%$ amino acid differences in ORF1, 0.4-4.1\% nucleotides and $0.9-6.2 \%$ amino acids in ORF2, and $0.5-3.3 \%$ nucleotides and $1-4.9 \%$ amino acids in ORF3). Interestingly, the Gll.17 capsid sequences of the two genetic subclades differed markedly from the oldest Gll.17 capsid sequence available in GenBank database, dating back to 1978 (23.3-24.8\% nucleotide and $14.2-16.6 \%$ amino acid differences in ORF2, and $19.4-27 \%$ nucleotide and $22.1-22.9 \%$ amino acid differences in $\mathrm{ORF}_{3}$ ).

Several changes in the VP1 sequence were observed between the two Kawasaki 2014 subclades, mostly, but not exclusively, affecting the antibody blockade sites, i.e. the putative epitopes (A-E) located in the capsid protruding hypervariable $\mathrm{P}_{2}$ domain (Figure 2). In the 543 amino acid VP1 protein, 17 amino acid changes (3.1\% divergence) and four insertions separate the two Kawasaki 2014 subclades, while 38 amino acid changes ( $7 \%$ divergence) and several insertions/ deletions separate the Kawasaki 2014 GII.17 NoV and the former Gll.17 recombinant forms.

\section{Discussion}

NoVs are a major cause of acute gastroenteritis in both children and adults, with sporadic cases and outbreaks in various epidemiological settings [6]. Although more than 30 cap genotypes within genogroups GI, GII, and GIV may infect humans [7], a single genotype, GII.4, has been associated since the mid-1990s with the majority (ca $70-80 \%$ ) of NoV-associated cases of gastroenteritis worldwide [12]. Gll.4 NoV strains undergo a continuous process of genetic/antigenic diversification and periodically generate new strains via accumulation of point mutations or recombination, with one novel GII.4 variant emerging every two to three years $[12,13]$ and becoming predominant globally. NoV vaccines based on GII.4 NoV strains are currently under development [14].

In the winter season 2014/15, the GII.P17-GII.17 NoV strain Kawasaki 2014 emerged in Asia, replacing the previously dominant Gll.4 genotype Sydney 2012 variant [1-4]. A signature of the Kawasaki 2014 variant is a novel pol type GII.P17, combined with a GII.17 ORF2 gene. Previously, NoVs with a GII.17 cap genotype possessed a GII.P4, GII.P3, GII.P13 or GII.P16 pol genotype [15-18]. Although being predominant in several Asian countries, this novel GII.P17-GII.17 strain has been detected in a limited number of cases on other continents [1-5]. The epidemiological trends exhibited by the Kawasaki 2014 NoV variant are considered unique, as, so far, this is the only non-GIl.4 NoV strain to have shown such epidemic pattern. The emergence of the novel GII.P17-GII.17 NoV strain in the Asian countries has been associated with increased NoV activity, i.e. with increased incidence of NoV-induced acute gastroenteritis, in the $2014 / 15$ winter season, compared to the previous (2013/14) winter season [1-3]. This pattern has been observed, but not consistently, during the worldwide spread of NoV Gll.4 variants [19]. Based on current literature on GII.17 NoVs, there is no indication on the clinical severity of the novel Gll.17 virus [1-5]. Likewise, our study did not assess whether Kawasaki 2014 NoVs are associated with increased severity of the clinical symptoms.

Hospital-based surveillance for NoV identified the emergence of GII.P17-GII.17 strains in Italy at the end of the 2014/15 winter season, in February 2015. The viruses were genetically closely related to GII.17 NoVs identified in the US and Asia in 2014 and 2015 [3,5], forming a distinct subclade of the Kawasaki 2014 GII.17 NoV variant. Co-circulation of two subclades of Kawasaki 2014 Gll.17 NoV with several amino acid changes in the putative capsid epitopes could suggest that this novel strain is undergoing fast diversification, mirroring what was seen globally for the epidemic GII.4 variants [12].

In addition, the emergence and spread of the novel Gll.17 variant Kawasaki 2014 could represent a challenge for the efficacy of the candidate NoV vaccines [14], that target the globally predominant GII.4 NoV, as it is not known whether vaccine immunity elicited to GII.4 NoV is cross-reactive with Gll.17 viruses. Continuous monitoring of the epidemiology of human NoV is important to understand the evolution of NoV and to implement the future strategies on NoV vaccines.

\section{Acknowledgements}

This study was partly supported by the projects 'Epidemiologia molecolare e studio dei meccanismi evolutivi di norovirus e rotavirus umani', granted by the University of Parma, Italy (Fondi di Ateneo 2014) and 'Norovirus: caratterizzazione molecolare ed epidemiologia', granted by the University of Palermo, Italy (Fondi di Ateneo 2012). 


\section{Conflict of interest}

None declared.

\section{Authors' contributions}

Conceived and designed the experiments: MCM, FT, VM; analysis of samples: MCM, FT, MC, GMG, SDG, VM; analysed and interpreted the data: MCM, FT, VM; wrote the manuscript: MCM, FT, VM; critical revision of the manuscript: $A C$, MC, GMG, SDG, MCA, FDC, CC; approved the final version: MCM, FT, AC, MC, GMG, SDG, MCA, FDC, CC, VM

\section{References}

1. Ful, Ail, JinM, JiangC, Zhangl, ShiC, et al. Emergence of a new GIl.17 norovirus variant in patients with acute gastroenteritis in Jiangsu, China, September 2014 to March 2015. Euro Surveill. 2015;20(24):21157. DOI: 10.2807/1560-7917.ES2015.20.24.21157 PMID: 26111236

2. LuJ, SunL, FangL, YangF, MoY, LaoJ, et al. Gastroenteritis Outbreaks Caused by Norovirus Gll.17, Guangdong Province, China, 2014-2015. Emerg Infect Dis. 2015;21(7):1240-2. DOI: 10.3201/eid2107.150226 PMID: 26080037

3. MatsushimaY, IshikawaM, ShimizuT, KomaneA, KasuoS, ShinoharaM, et al. Genetic analyses of Gll.17 norovirus strains in diarrheal disease outbreaks from December 2014 to March 2015 in Japan reveal a novel polymerase sequence and amino acid substitutions in the capsid region. Euro Surveill. 2015;20(26):21173. DOI: 10.2807/1560-7917. ES2015.20.26.21173 PMID: 26159307

4. de GraafM, van BeekJ, VennemaH, PodkolzinAT, Hewitt), BucardoF, et al. Emergence of a novel GII.17 norovirus - End of the GIl.4 era? Euro Surveill. 2015;20(26):21178. DOI: 10.2807/1560-7917.ES2015.20.26.21178 PMID: 26159308

5. ParraGI, GreenKY. Genome of Emerging Norovirus GII.17, United States, 2014.Emerg Infect Dis. 2015;21(8):1477-9. DOI: 10.3201/ eid2108.150652 PMID: 26196235

6. Green KY. 2013. Caliciviridae: the noroviruses. In: Knipe, D.M., Howley, P.M. (Eds.), Fields virology, 6th ed. Wolters Kluwer Health/Lippincott Williams and Wilkins, Philadelphia, pp. 949-9.

7. KronemanA, VegaE, VennemaH, VinjéJ, WhitePA, HansmanG, et al. Proposal for a unified norovirus nomenclature and genotyping. Arch Virol. 2013;158(10):2059-68. DOI: 10.1007/ S00705-013-1708-5 PMID: 23615870

8. Dutch Ministry of Health. Welfare and Sport. National Institute for Public Health and the Environment (RIVM). Norovirus Genotyping Tool Version 1.o. Bilthoven: RIVM. [Accessed 24 Aug 2015]. Available from: http://www.rivm.nl/mpf/norovirus/ typingtool

9. WangQH, HanMG, CheethamS, SouzaM, FunkJA, SaifLJ. Porcine noroviruses related to human noroviruses. Emerg Infect Dis. 2005;11(12):1874-81. DOI: 10.3201/eid1112.050485 PMID: 16485473

10. VennemaH, de BruinE, KoopmansM. Rational optimization of generic primers used for Norwalk-like virus detection by reverse transcriptase polymerase chain reaction.J Clin Virol. 2002;25(2):233-5. DOI: 10.1016/S1386-6532(02)00126-9 PMID: 12367660

11. TamuraK, StecherG, PetersonD, FilipskiA, KumarS, MEGA6: Molecular Evolutionary Genetics Analysis version 6.o.Mol Biol Evol. 2013;30(12):2725-9. DOI: 10.1093/molbev/mst197 PMID: 24132122

12. Hoa TranTN, TrainorE, NakagomiT, CunliffeNA, NakagomiO. Molecular epidemiology of noroviruses associated with acute sporadic gastroenteritis in children: global distribution of genogroups, genotypes and GII.4 variants.J Clin Virol. 2013;56(3):269-77. DOI: 10.1016/j.jcv.2012.11.011 PMID: 23218993

13. SiebengaJJ, VennemaH, ZhengDP, VinjéJ, LeeBE, PangXL, et al. Norovirus illness is a global problem: emergence and spread of norovirus GII.4 variants, 2001-2007. J Infect Dis. 2009;200(5):802-12. DOI: 10.1086/605127 PMID: 19627248

14. BernsteinDI, AtmarRL, LyonGM, TreanorJJ, ChenWH, JiangX, et al. Norovirus vaccine against experimental human GIl. 4 virus illness: a challenge study in healthy adults. J Infect Dis. 2015;211(6):870-8. DOI: 10.1093/infdis/jiu497 PMID: 25210140

15. AyukekbongJA, FobisongC, TahF, LindhM, Nkuo-AkenjiT, BergströmT. Pattern of circulation of norovirus Gll strains during natural infection.J Clin Microbiol. 2014;52(12):4253-9. DOI: 10.1128/JCM.01896-14 PMID: 25274996

16. Mans), MurrayTY, TaylorMB. Novel norovirus recombinants detected in South Africa.Virol J. 2014;11(1):168. DOI: 10.1186/1743-422X-11-168 PMID: 25228444

17. RackoffLA, BokK, GreenKY, KapikianAZ. Epidemiology and evolution of rotaviruses and noroviruses from an archival WHO Global Study in Children (1976-79) with implications for vaccine design.PLoS ONE. 2013;8(3):e59394. DOI: 10.1371/journal. pone.0059394 PMID: 23536875

18. WangYH, ZhouDJ, ZhouX, YangT, GhoshS, PangBB, et al. Molecular epidemiology of noroviruses in children and adults with acute gastroenteritis in Wuhan, China, 2007-2010. Arch Virol. 2012;157(12):2417-24. DOI: 10.1007/s00705-012-1437-1 PMID: 22886184

19. VegaE, BarclayL, GregoricusN, ShirleySH, LeeD, VinjéJ. Genotypic and epidemiologic trends of norovirus outbreaks in the United States, 2009 to 2013.J Clin Microbiol. 2014;52(1):147-55. DOI: 10.1128/JCM.02680-13 PMID: 24172151 